\title{
Effective transport properties of composites with a doubly-periodic array of fiber pairs and with a triangular array of fibers
}

\author{
P. Yan ${ }^{1, *}$, Z. A. Zhang ${ }^{1}$, F. L. Chen ${ }^{2}$, C. P. Jiang ${ }^{1,3}$, X. J. Wang ${ }^{1}$, and Z. P. Qiu ${ }^{1, *}$ \\ ${ }^{1}$ School of Aeronautic Science and Engineering, Beihang University (BUAA), Beijing 100191, China \\ ${ }^{2}$ AVIC Academy of Aeronautic Propulsion Technology, Beijing 101300, China \\ ${ }^{3}$ State Key Laboratory of Nonlinear Mechanics (LNM), Institute of Mechanics, Chinese Academy of Sciences, Beijing \\ 100190, China
}

Received 13 March 2017, revised 3 August 2017, accepted 28 August 2017

Published online 2 October 2017

Key words Composites, fiber pairs, triangular array, effective transport properties, complex variable method.

\begin{abstract}
This paper presents a complex variable solution for the effective transport properties of composites with a doubly-periodic array of fiber pairs. By using the centrosymmetry of the problem, the method of Rayleigh and Natanzon-Filshtinsky's approach can be simply extended to the problems with two fibers per unit cell. The infinite system constructed in this paper only slightly complicates Rayleigh's system for the problems with one fiber per unit cell. Approximate analytical formulae of the effective transport properties for different fiber-pair arrays are obtained. The influence of pairwise interaction in fiber pairs on the effective transport properties is discussed in the numerical examples. As a special case of a doubly-periodic array of fiber pairs, effective transport property of composites with a triangular array of fibers is obtained. The obtained approximate analytical formulae are written in a concise form with good accuracy, thus are convenient for engineering application in most cases, except for those approaching the limit case of percolation when the perfectly conducting fibers become touching. Besides the square array and hexagonal array, the triangular fiber array (similar to carbon atom arrangement in graphene) is another special symmetric fiber array which results into transversely isotropic effective property. Therefore, the present solution for the triangular array is an extension of those for the square array and hexagonal array. The comparison of the results for the three symmetric fiber arrays reveals that the triangular fiber array has the highest conductivity. In addition, accuracy of the present solution is analyzed in the numerical examples.
\end{abstract}

(C) 2017 WILEY-VCH Verlag GmbH \& Co. KGaA, Weinheim

\section{Introduction}

Effective properties of composites play an important role in the design of composite structures in applications. In particular, effective transport properties such as thermal conductivity, dielectric constant and fluid permeability, as well as their mathematically analogous properties such as elastic modulus, are key parameters characterizing the macroscopic characteristics of composites [46]. The problems of predicting the effective transport properties of composites from their microstructure are valuable for designing and evaluation of composites, so they have attracted a lot of researchers $[29,30,35,46]$. Based on their research works, the main framework for solving such a problem has been established. For example, numerical methods including FEM are outlined in [30], and various numerical and analytical methods based on the multipoint correlation functions are discussed in [46]. However, analytical solutions are still useful in disclosing the inherent law of the relation of effective transport properties to microstructures. Additionally, analytical methods are more effective in solving some specific problems involving imperfect interface [24], interface cracks [47] or two approaching inclusions [28], because it needs particular tricks for numerical methods to obtain accurate results of fields with high gradient or singularity at hot spots.

As for fiber-reinforced composites, or more generally an array of cylinders in matrix, fiber/cylinder distribution is one of the key factors which influence the effective transport properties. Among all the analytical solutions, the classical MaxwellGarnett estimation [29] is valid for dilute composites. This estimations are based on a model of one fiber embedded in infinite matrix, thus are without parameters reflecting fiber distribution. As a result, this model cannot consider properly the fiber interaction, especially at a high fiber volume fraction. It can be proved that this model gives a lower bound of effective transport property [18]. Another kind of analytical solutions is based on a composite model with fibers in a regular array, such as square array, hexagonal array, rectangular array, rhombic array and general periodic array. Perrins et al. [36] and McPhedran et al. [27] modified and extended Rayleigh's solution [38] for square array and hexagonal array. Mityushev et al.

* $\quad$ Corresponding author: E-mail: zpqiu@buaa.edu.cn (Z.P. Qiu); yanpeng117@buaa.edu.cn (P. Yan). Tel.: +86 01082317507 
$[11,13,32]$ applied the method of functional equations to the square array and hexagonal array, and discussed two extreme cases of porous materials and superconducting cylinders. Rylko [42] and Mityushev [33] also applied this method to the cases of rectangular array of cylinders. Castro et al. [3] applied the method of functional equations [5] to the cases of square array with imperfect interface between matrix and inclusions. Jiang et al. [21] presented a solution for general doublyperiodic array by using Eshelby's equivalent inclusion concept integrated with the results from the doubly quasi-periodic Riemann boundary value problems. Rodriguez-Ramos et al. [16, 17, 26, 40,41] applied an asymptotic homogenization method (AHM) to solve the anti-plane elastic modulus, plane elastic modulus and the piezoelectric modulus for the cases of arbitrary doubly-periodic arrays. Godin [15] developed a method based on the use of elliptic function for the cases of general doubly-periodic arrays. Andrianov and Mityushev [1] pointed out that this method is related to the method of Natanzon-Filshtinsky for conductivity problems [6,7]. The authors also developed an eigenfunction expansion-variational method (EEVM) [51] and a series method [50] extended from Rayleigh's solution to solve the effective conductivity for general periodic arrays, in order to obtain approximate analytical formulae with high accuracy and in a concise form. As all above models are with uniform regular fiber distributions, the influence of fiber clustering was not considered.

Fiber \particle clusters are common in composites [30]. The influence of Fiber \particle clustering was investigated in existing works $[10,30,44]$ by using random microstructure model. A fiber pair can be seen as the simplest fiber cluster containing two fibers, so the model of fiber pair can be used to investigate the influence of fiber clustering to certain extent. McPhedran et al. [28] and Radchik et al. [37] discussed the cases of a pair of fibers in infinite matrix. Reuben et al. [39] discussed the cases of a chain of fiber pairs in infinite matrix. Rylko [43] applied the method of functional equations to calculate the conductivity of a rectangular array of cylinder pairs and discussed the effect of polydispersity. To the best of our knowledge, solution for the case of a general doubly-periodic array of fiber pairs has not been reported. This motivated us to extend the complex variable solution [50] for array of sole fibers to a general doubly-periodic array of fiber pairs. The present complex variable method [50] is expressed in a series form and based on the elliptic function theory, thus, which is related to other solutions based on the elliptic function theory, such as the exact method of functional equations by Mityushev et al. [11, 13,32,33,42], and the solutions by Jiang et al. [21] and by Godin [15]. When the series is truncated to finite order, an approximate solution is obtained.

It is worth mentioning that Mityushev and Gluzman [12,14,34] presented a universal method of functional equations for more general cases that a unit cell contains any number of randomly-distributed fibers (disks). Recently, Andrianov and Mityushev [1] outlined the method of functional equations, as well as the above-mentioned methods of Rayleigh, NatanzonFilshtinsky, and asymptotic homogenization approaches. They pointed out that the method of functional equations stands out against other methods in exact solution to 2D problems with one circular inclusion per cell. This method in discrete form is equivalent to the series method that was demonstrated in [42]. When one applies this method to calculate effective conductivities of composites with doubly-periodic array of fibers, the same problem occurs as it does in the series method [50], that is, the sum $S_{2}$ used by Eq. (13) in [50]) is conditionally convergent. The reason is that the value of the sum $S_{2}$ is dependent on the summation sequence. For symmetric fiber arrays (such as hexagonal array, square array, rhombic array and rectangular array), the sum $S_{2}$ can be expressed by the Eisenstein summation (defined by Eq. (5.105) in [32] and denoted differently by $S_{2}^{*}$ in [50]) and then can be calculated by the Eq. (5.107) in [32] (or Eq. (23') in [43]). However, for general doubly-periodic arrays without symmetry, the sum $S_{2}$ cannot be simply expressed by the Eisenstein summation, but it should be eliminated by using a supplementary equation [50]. Therefore, the problem of calculating the effective conductivity of composites with a general doubly-periodic array of multiple fibers still remains to be solved.

In addition, as a special case of a doubly-periodic array of fiber pairs, a triangular array of sole fibers can be seen as a hexagonal array of special fiber pairs. Besides square array [27] and hexagonal array [36], triangular fiber array (similar to carbon atom arrangement in graphene) is another special symmetric fiber array which results into transversely isotropic effective property. Therefore, triangular fiber array is of interest in engineering application. For example, this kind of special symmetric array appears in 2D photonic crystals [8,22], porous medium model [4] and nuclear reactor [2]. From hexagonal array to square array to triangular array, the limit volume fraction of fibers decreases. Therefore, solving the transport problem of composites with triangular array of fibers is helpful to investigate the influence of fiber-distribution uniformity on effective transport properties. Additionally, all fibers are equal in the triangular fiber array. This nature makes it possible to derive concise approximate analytical formulae of effective transport properties, as it does for hexagonal fiber array and square fiber array [50]. Concise analytical formulae, as we know, are convenient for engineering application. However, approximate analytical formulae or even numerical results of effective transport properties for triangular fiber array have not been reported. This also motivated us to carry out the present work.

Without loss of generality, the effective transport properties discussed here refer to thermal conductivities. This paper is organized as follows: In Sect. 2, basic equations of the problem are formulated in complex variables, and then the complex potential in matrix contains a doubly-periodic array of fiber pairs is constructed and expanded into Laurent series. In Sect. 3, calculation of two kinds of summations involved in the expansion of the complex potential is introduced. In Sect. 4, fiber-matrix interface condition and unit-cell boundary condition of the problem are introduced, through which 
linear equations about the unknown expansion coefficients are derived. In Sect. 5, effective conductivities are calculated by average field method, also a series of approximate analytical formulae of effective conductivities are given for different fiber-pair arrays including the triangular fiber array. In Sect. 6, numerical examples are taken to verify the validity and accuracy of the present solution, and to investigate the influence of pairwise interaction in fiber pairs. Finally, numerical results of effective conductivities for the triangular fiber array are given and compared in Sect. 6.4.

\section{Basic equations and the complex potential in matrix}

Consider a composite with a doubly-periodic array of fiber pairs, whose transverse section is shown in Fig. 1. Each fiber is of radius $R$. The doubly-periodic array of fiber pairs can be seen as two identical doubly-periodic arrays of fibers generated by translating one doubly-periodic array of fibers by a complex translation $\delta$. Two fundamental complex periods of the array are denoted by $\omega_{1}$ and $\omega_{2}$, and then an arbitrary complex period $\omega_{r s}$ of the doubly-periodic array can be expressed by

$$
\omega_{r s}=r \omega_{1}+s \omega_{2},
$$

where $r$ and $s$ are integers. Without loss of generality, unit area is assumed for the fundamental unit cell, that is

$$
\frac{1}{2 \mathrm{i}}\left(\bar{\omega}_{1} \omega_{2}-\omega_{1} \bar{\omega}_{2}\right)=1
$$

The fibers have transversely isotropic conductivity and are placed in a matrix of isotropic conductivity. For steady-state heat conduction in a media with isotropic conductivity $k$, the in-plane temperature field satisfies Laplace's equation [20]. The temperature $T$, heat flux components $\left\{q_{1}, q_{2}\right\}$ and heat transfer rate $\Phi$ can be formulated by a complex potential $f(z)$ and its derivative:

$$
\begin{aligned}
& q_{1}-\mathrm{i} q_{2}=-k f^{\prime}(z), \\
& T=\frac{1}{2}[f(z)+\overline{f(z)}], \\
& \Phi=\frac{-k}{2 \mathrm{i}}[f(z)-\overline{f(z)}]_{\mathrm{A}}^{\mathrm{B}},
\end{aligned}
$$

where $\mathrm{i}^{2}=-1, z=x_{1}+\mathrm{i} x_{2}$ is a complex variable, the over bar denotes the complex conjugate, the prime denotes the derivative with respect to $z,[\cdot]_{\mathrm{A}}^{\mathrm{B}}$ denotes the difference of the values of the bracketed function from point A to point $\mathrm{B}$.

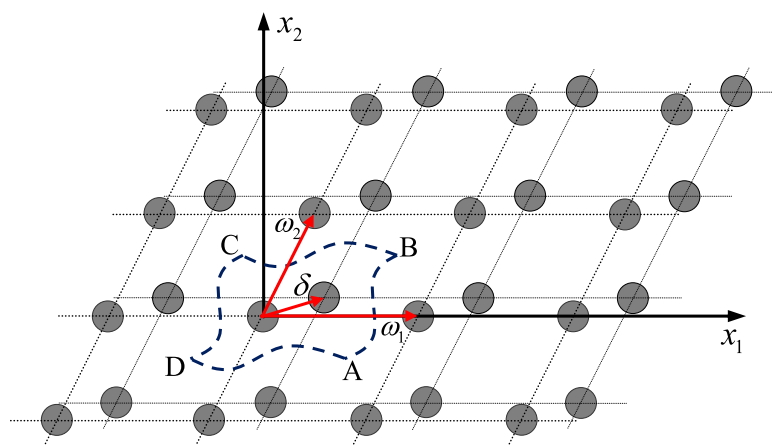

Fig. 1 Transverse section of a composite with a doubly-periodic array of fiber pairs. ABCD is aunit cell of arbitrary shape containinga fiber pair. $\omega_{1}$ and $\omega_{2}$ are two fundamental complex periods. $\delta$ is a complex translation from one fiber to another in the fiber pair.

The complex potential in each circular fiber region can be expressed in a Taylor series [51]. The matrix region surrounding fibers are multiply connected, in which the complex potential will be constructed by using superposition method in the following. The temperature, gradient and flux expressed by the constructed potential and its derivative should satisfy the quasi-periodicity/periodicity condition, fiber-matrix interface condition and the boundary condition of prescribed external gradient. 
If there is no fiber in matrix, the internal gradient in the matrix subjected to the external gradient $H_{j}^{0}(j=1,2)$ should be uniform. Therefore the complex potential $f_{\mathrm{m}}(z)$ in the matrix contains fibers should include two parts:

$$
f_{\mathrm{m}}(z)=A_{0} z+f_{\mathrm{m} 0}(z),
$$

where the first part is corresponding to the uniform internal gradient results from the external gradient, that is

$$
A_{0}=H_{1}^{0}-\mathrm{i} H_{2}^{0} \text {. }
$$

The second part in (4) is induced by the appearance of the two doubly-periodic arrays of fibers as depicted in Fig. 1, so it can be divided into two parts:

$$
f_{\mathrm{m} 0}(z)=f_{\mathrm{m} 01}(z)+f_{\mathrm{m} 02}(z-\delta),
$$

where $f_{\mathrm{m} 01}(z)$ and $f_{\mathrm{m} 02}(z-\delta)$ are, respectively, induced by the appearance of the fibers located at $\omega_{r s}$ and $\omega_{r s}+\delta$. Due to the centrosymmetry of the problem about the point located at $\delta / 2$, the derivatives of $f_{\mathrm{m} 01}(z)$ and $f_{\mathrm{m} 02}(z)$ satisfy $f_{\mathrm{m} 01}^{\prime}(z)=f_{\mathrm{m} 02}^{\prime}(-z)$. The constants in $f_{\mathrm{m} 01}(z)$ and $f_{\mathrm{m} 02}(z)$ do not influence the gradient, thus can be neglected, then it can be obtained that

$$
f_{\mathrm{m} 02}(z)=-f_{\mathrm{m} 01}(-z)
$$

Therefore, Eq. (6) can be rewritten as

$$
f_{\mathrm{m} 0}(z)=f_{\mathrm{m} 01}(z)-f_{\mathrm{m} 01}(-z+\delta) .
$$

Due to the periodicity with periods $\omega_{r s}$ of the fiber array, $f_{\mathrm{m} 01}(z)$ can be expressed by a summation

$$
f_{\mathrm{m} 01}(z)=f_{0}(z)+\sum_{r, s}^{\prime} f_{0}\left(z-\omega_{r s}\right)
$$

where $\sum_{r, s}^{\prime}$ means the sum taken over all $r^{2}+s^{2} \neq 0$, the same below. Then the second part in (4) can be expressed by a summation:

$$
f_{\mathrm{m} 0}(z)=f_{0}(z)-f_{0}(-z+\delta)+\sum_{r, s}^{\prime}\left[f_{0}\left(z-\omega_{r s}\right)-f_{0}\left(-z+\omega_{r s}+\delta\right)\right] .
$$

The complex potential $f_{0}\left(z-\omega_{r s}\right)$ is induced by the appearance of the fiber located at $\omega_{r s}$, which approaches 0 when $z-\omega_{r s} \rightarrow \infty$. Thus $f_{0}(z)$ can be expanded as follows:

$$
f_{0}(z)=\sum_{n=1}^{\infty} A_{n} \frac{1}{z^{n}}
$$

where $A_{n}$ are the unknown expansion coefficients. Therefore

$$
f_{\mathrm{m} 0}(z)=\sum_{n=1}^{\infty} A_{n} \frac{1}{z^{n}}-\sum_{n=1}^{\infty} A_{n} \frac{1}{(-z+\delta)^{n}}+\sum_{r, s},\left[\sum_{n=1}^{\infty} A_{n} \frac{1}{\left(z-\omega_{r s}\right)^{n}}-\sum_{n=1}^{\infty} A_{n} \frac{1}{\left(-z+\omega_{r s}+\delta\right)^{n}}\right] .
$$

Note that

$$
\begin{aligned}
\frac{1}{\left(z-\omega_{r s}\right)^{n}} & =\frac{1}{\left(-\omega_{r s}\right)^{n}}+\sum_{j=1}^{\infty}(-1)^{j} \mathrm{C}_{n+j-1}^{j} \frac{1}{\left(-\omega_{r s}\right)^{n+j}} z^{j}, \\
\frac{1}{(-z+\delta)^{n}} & =\frac{1}{\delta^{n}}+\sum_{j=1}^{\infty} \mathrm{C}_{n+j-1}^{j} \frac{1}{\delta^{n+j}} z^{j} \\
\frac{1}{\left(-z+\delta+\omega_{r s}\right)^{n}} & =\frac{1}{\left(\delta+\omega_{r s}\right)^{n}}+\sum_{j=1}^{\infty} \mathrm{C}_{n+j-1}^{j} \frac{1}{\left(\delta+\omega_{r s}\right)^{n+j}} z^{j}
\end{aligned}
$$

with

$$
\mathrm{C}_{n+j-1}^{j}=\frac{(n+j-1) !}{j !(n-1) !}
$$


Then Eq. (12) can be expanded as

$$
\begin{aligned}
f_{\mathrm{m} 0}(z)= & \sum_{n=1}^{\infty} A_{n}\left(\frac{1}{z}\right)^{n}+\sum_{n=1}^{\infty} A_{n} \sum_{j=1}^{\infty} \mathrm{C}_{n+j-1}^{j}\left[-\frac{1}{\delta^{n+j}}+(-1)^{j} \sum_{r, s}^{\prime} \frac{1}{\left(-\omega_{r s}\right)^{n+j}}-\sum_{r, s}^{\prime} \frac{1}{\left(\delta+\omega_{r s}\right)^{n+j}}\right] z^{j} \\
& +\sum_{n=1}^{\infty} A_{n}\left[-\frac{1}{\delta^{n}}+\sum_{r, s}^{\prime} \frac{1}{\left(-\omega_{r s}\right)^{n}}-\sum_{r, s}^{\prime} \frac{1}{\left(\delta+\omega_{r s}\right)^{n}}\right] .
\end{aligned}
$$

After neglecting the constant, Eq. (15) can be rewritten as

$$
f_{\mathrm{m} 0}(z)=\sum_{n=1}^{\infty} A_{n} \frac{1}{z^{n}}+\sum_{n=1}^{\infty} A_{n} \sum_{j=1}^{\infty} \mathrm{C}_{n+j-1}^{j}\left[(-1)^{n} S_{n+j}-E_{n+j}(\delta)\right] z^{j}
$$

with

$$
\begin{aligned}
& S_{k}=S_{k}\left(\omega_{1}, \omega_{2}\right)=\sum_{r, s}{ }^{\prime} \frac{1}{\omega_{r s}^{k}}, k \geq 2, \\
& E_{k}(\delta)=E_{k}\left(\omega_{1}, \omega_{2} ; \delta\right)=\frac{1}{\delta^{k}}+\sum_{r, s}{ }^{\prime} \frac{1}{\left(\delta+\omega_{r s}\right)^{k}}, k \geq 2 .
\end{aligned}
$$

The calculations of the two summations will be discussed in Sect. 3. Note that the summation used here is different to the Eisentein summation [48], because the summation sequence is not given. However, when $k \geq 3$, they are equal, because in these cases the summation is absolutely convergent and independent to the summation sequence [25].

With Eq. (16), the complex potential in matrix (4) can be written in the following Laurent series:

$$
f_{\mathrm{m}}(z)=\sum_{n=1}^{\infty} A_{n} \frac{1}{z^{n}}+\sum_{n=1}^{\infty}\left\{A_{0} \delta_{1, n}+\sum_{j=1}^{\infty} A_{j} \mathrm{C}_{j+n-1}^{n}\left[(-1)^{j} S_{j+n}-E_{j+n}(\delta)\right]\right\} \cdot z^{n},
$$

where $\delta_{1, n}$ is the Kronecker delta symbol, being unity for $n=1$ and zero otherwise.

The constructed complex potential $f_{\mathrm{m}}(z)$ expressed by Eqs. (4) and (12) is quasi-periodic, which will be proved in Sect. 4.4. Therefore, the temperature gradient and heat flux fields expressed by its derivative satisfy the doubly-periodicity conditions. The unknown coefficients in the Laurent expansion (19) of the complex potential will be determined by the interface condition between fiber and matrix, and the unit-cell boundary condition in Sect. 4.

\section{Calculation of the summations $S_{k}$ and $E_{k}(\delta)$}

The calculation of the sum $S_{k}$ defined by (17) are detailed in the references [19,31], as well as in the previous paper [50]. Note that $S_{2}$ is conditionally convergent, and $S_{2 k+1}=0$ for $k \geq 1$.

Now let's calculate the summation $E_{k}(\delta)$ defined by (18). When $k=2, E_{2}(\delta)$ is also conditionally convergent, which is related to the Weierstrass $\wp$ function [19]:

$$
\wp(\delta)=\wp\left(\omega_{1}, \omega_{2} ; \delta\right)=\frac{1}{\delta^{2}}+\sum_{r, s},\left[\frac{1}{\left(\delta-\omega_{r s}\right)^{2}}-\frac{1}{\omega_{r s}^{2}}\right] .
$$

That is

$$
\wp(\delta)=\wp(-\delta)=E_{2}(\delta)-S_{2} .
$$

When $k \geq 3, E_{k}(\delta)$ is absolutely convergent. From (18) and (20), we have

$$
E_{k}(\delta)=(-1)^{k-2} \frac{1}{(k-1) !} \wp^{(k-2)}(\delta) \text { for } k \geq 3 \text {, }
$$

where

$$
\wp^{\prime}(\delta)=\frac{\mathrm{d} \wp(\delta)}{\mathrm{d} \delta}, \quad \wp^{\prime \prime}(\delta)=\frac{\mathrm{d}^{2} \wp(\delta)}{\mathrm{d} \delta^{2}}, \quad \wp^{\prime \prime \prime}(\delta)=\frac{\mathrm{d}^{3} \wp(\delta)}{\mathrm{d} \delta^{3}}, \quad \wp^{(k-2)}(\delta)=\frac{\mathrm{d}^{(k-2)} \wp(\delta)}{\mathrm{d} \delta^{(k-2)}},
$$

and $(k-1) !=1 \times 2 \times 3 \times \cdots \times(k-2) \times(k-1)$. 
Note that there exists the following equation [25]:

$$
\wp^{\prime 2}(\delta)=4 \wp^{3}(\delta)-60 S_{4} \wp(\delta)-140 S_{6} .
$$

Through deriving it, we have

$$
\wp^{\prime \prime}(\delta)=6 \wp^{2}(\delta)-30 S_{4},
$$

and the following recursive formulae:

$$
\wp^{(k)}(\delta)=12 \sum_{j=0}^{k-3} \mathrm{C}_{k-3}^{j} \wp^{(k-3-j)}(\delta) \wp^{(j+1)}(\delta) \quad \text { for } \quad k \geq 3 .
$$

Then from (22) and (26), $E_{k}(\delta)(k \geq 3)$ can eventually be expressed by $\wp(\delta)$ and $\wp^{\prime}(\delta)$. The first four expressions are as follows:

$$
\begin{aligned}
& E_{3}(\delta)=-\frac{1}{2} \wp^{\prime}(\delta), \\
& E_{4}(\delta)=\frac{1}{6} \wp^{\prime \prime}(\delta)=\wp^{2}(\delta)-5 S_{4}, \\
& E_{5}(\delta)=-\frac{1}{12} \wp^{\prime \prime \prime}(\delta)=-\wp(\delta) \wp^{\prime}(\delta), \\
& E_{6}(\delta)=\frac{1}{60} \wp^{(4)}(\delta)=2 \wp^{3}(\delta)-18 S_{4} \wp(\delta)-28 S_{6} .
\end{aligned}
$$

The values of the two elliptic functions $\wp(\delta)$ and $\wp^{\prime}(\delta)$ can be calculated directly in mathematical software Mathematica [49], or from their Fourier Expansions [25].

\section{Interface condition and boundary condition}

\subsection{Interface condition}

Heat transfer rate $\Phi$ and temperature $T$ expressed by the constructed complex potential and its derivative, should also satisfy the fiber-matrix interface condition. For the case of perfect interface, the heat transfer rate and temperature across the interface are continuous:

$$
\Phi_{\mathrm{f}}=\Phi_{\mathrm{m}}, \quad T_{\mathrm{f}}=T_{\mathrm{m}}, \quad \text { at } \quad|z|=R,
$$

where the subscripts " $\mathrm{m}$ " and "f" denote matrix and fiber, respectively. By substituting the complex potentials into (3), and then into (28), the coefficient equations are obtained as [51]:

$$
\bar{A}_{n}=\eta_{n} R^{2 n}\left\{A_{0} \delta_{1, n}+\sum_{j=1}^{\infty} A_{j} \mathrm{C}_{j+n-1}^{n}\left[(-1)^{j} S_{j+n}-E_{j+n}(\delta)\right]\right\}, n=1,2,3 \ldots,
$$

where $\eta_{n}$ is a constant equal to $\left(k_{\mathrm{m}}-k_{\mathrm{f}}\right) /\left(k_{\mathrm{m}}+k_{\mathrm{f}}\right)$ for perfect interface. $k_{\mathrm{f}}$ and $k_{\mathrm{m}}$ are the conductivities of fiber and matrix, respectively. Note that the present problem is not centrosymmetric about the origin, thus both the odd terms and even terms exist, which is different from Eq. (15) in Yan et al. [50].

For the cases of contact resistance interface and coating interface between fibers and matrix, Eq. (29) is the same, but $\eta_{n}$ is not a constant, which is given similarly in Yan et al. [50].

\subsection{Boundary condition of prescribed external gradient}

Because the coefficient equations (29) involve the conditionally convergent sums $S_{2}$ and $E_{2}(\delta)$, the expansion coefficients cannot be determined by now. Thus, a supplementary equation is needed.

The complex potential $f_{\mathrm{m}}(z)$ constructed in Sect. 2 is for the matrix in a composite under a prescribed external gradient $H_{j}^{0}$, so its expansion coefficients in (19) must satisfy this boundary condition. Due to the periodicity, average gradient $\left\langle H_{j}\right\rangle$ over any unit cell should be equal to the average gradient over the whole transverse section of the composite, and that should be equal to the prescribed external gradient $H_{j}^{0}$. Therefore, considering Eq. (5), we have

$$
\left\langle H_{1}\right\rangle+\mathrm{i}\left\langle H_{2}\right\rangle=H_{1}^{0}+\mathrm{i} H_{2}^{0}=\bar{A}_{0} .
$$


Equation (30) is a supplementary equation to the coefficient equations (29), which makes the solution complete.

Before applying the supplementary equation (30), the average gradient $\left\langle H_{j}\right\rangle$ should be expressed by the coefficients in (19), which will be completed in the following.

\subsection{Relation of average gradient to average flux}

Consider an arbitrary unit cell ABCD containing a fiber pair as shown in Fig. 1. The area and the boundary of the unit cell are denoted by $V$ and $\partial V$, respectively. Without loss of generality, the boundaries of the unit cell are always assumed to be located at the matrix. The relation of the average gradient and average flux in complex form can be expressed by the complex potential in matrix [50]:

$$
\left\langle q_{1}\right\rangle+\mathrm{i}\left\langle q_{2}\right\rangle-k_{\mathrm{m}}\left(\left\langle H_{1}\right\rangle+\mathrm{i}\left\langle H_{2}\right\rangle\right)=\frac{k_{\mathrm{m}}}{V} \frac{\mathrm{i}}{2} \oint_{\partial V}\left[f_{\mathrm{m}}(z)-f_{\mathrm{m}}^{\prime}(z) \mathrm{z}\right] \mathrm{d} z
$$

where

$$
\left\langle q_{i}\right\rangle=\frac{1}{V} \int_{V} q_{i} \mathrm{~d} V, \quad\left\langle H_{i}\right\rangle=\frac{1}{V} \int_{V} H_{i} \mathrm{~d} V, \quad i=1,2 .
$$

From (4) and (12), and according to the Residue theorem,

$$
\oint_{\partial V}\left[f_{\mathrm{m}}(z)-f^{\prime}{ }_{\mathrm{m}}(z) \mathrm{z}\right] \mathrm{d} z=2 \pi \mathrm{i}\left\{\operatorname{Res}\left[f_{\mathrm{m}}(z)-{f^{\prime}}_{\mathrm{m}}(z) \mathrm{z}, 0\right]+\operatorname{Res}\left[f_{\mathrm{m}}(z)-f^{\prime}{ }_{\mathrm{m}}(z) \mathrm{z}, \delta\right]\right\}=2 \pi \mathrm{i} \cdot 4 A_{1} .
$$

If $V=1$, Eq. (31) can be rewritten as

$$
\left\langle q_{1}\right\rangle+\mathrm{i}\left\langle q_{2}\right\rangle-k_{\mathrm{m}}\left(\left\langle H_{1}\right\rangle+\mathrm{i}\left\langle H_{2}\right\rangle\right)=-k_{\mathrm{m}} \cdot 4 \pi A_{1} .
$$

The left side of (34) is the difference between the average fluxes over the unit cells with and without a fiber pair, under the same average gradient $\left\langle H_{j}\right\rangle$. It can be seen that such a difference is only related to the first coefficient $A_{1}$ in the coefficient equation (29).

\subsection{Average flux over a unit cell}

Consider again the unit cell ABCD of unit area in Fig. 1. Due to the periodicity, complex coordinates at the three corners A, B, and C satisfy

$$
z_{\mathrm{B}}-z_{\mathrm{A}}=\omega_{2}, \quad z_{\mathrm{C}}-z_{\mathrm{B}}=-\omega_{1},
$$

By application of the Green theorem and the periodicity of the flux field, the average flux over the unit cell in complex form can be expressed by

$$
\left\langle q_{1}\right\rangle+i\left\langle q_{2}\right\rangle=\Phi^{\mathrm{AB}} \omega_{1}+\Phi^{\mathrm{BC}} \omega_{2},
$$

where $\Phi^{\mathrm{AB}}$ and $\Phi^{\mathrm{BC}}$ are the heat transfer rate $\Phi$ across boundaries $\mathrm{AB}$ and BC, respectively. From Eq. ( $3 \mathrm{c}$ ), they can be calculated from the complex functions at the corners:

$$
\Phi^{\mathrm{AB}}=k_{\mathrm{m}} \operatorname{Im}\left[f_{\mathrm{m}}\left(z_{\mathrm{B}}\right)-f_{\mathrm{m}}\left(z_{\mathrm{A}}\right)\right], \quad \Phi^{\mathrm{BC}}=k_{\mathrm{m}} \operatorname{Im}\left[f_{\mathrm{m}}\left(z_{\mathrm{C}}\right)-f_{\mathrm{m}}\left(z_{\mathrm{B}}\right)\right] .
$$

According to the Weierstrass $\varsigma$ function [25]

$$
\zeta(z)=\zeta\left(\omega_{1}, \omega_{2} ; z\right)=\frac{1}{z}+\sum_{r, s}^{\prime}\left(\frac{1}{z-\omega_{r s}}+\frac{1}{\omega_{r s}}+\frac{z}{\omega_{r s}^{2}}\right),
$$

and Eq. (17a), we have

$$
\begin{aligned}
& \frac{1}{z}+\sum_{r, s}^{\prime} \frac{1}{z-\omega_{r s}}=\zeta(z)-\sum_{r, s}^{\prime} \frac{1}{\omega_{r s}}-S_{2} z, \\
& \frac{1}{z^{2}}+\sum_{r, s}^{\prime} \frac{1}{\left(z-\omega_{r s}\right)^{2}}=-\zeta^{\prime}(z)+S_{2}, \\
& \frac{1}{z^{n}}+\sum_{r, s}^{\prime} \frac{1}{\left(z-\omega_{r s}\right)^{n}}=(-1)^{n-1} \frac{1}{n !} \zeta^{(n-1)}(z), \quad n \geq 3 .
\end{aligned}
$$


From Eq. (39), the potential $f_{\mathrm{m}}(z)(4),(12)$ can be expressed by $\zeta(z)$ and its derivatives:

$$
\begin{aligned}
f_{\mathrm{m}}(z)= & A_{0} z+\sum_{n=1}^{\infty} A_{n} \frac{1}{(n-1) !}\left[(-1)^{n-1} \varsigma^{(n-1)}(z)+\varsigma^{(n-1)}(z-\delta)\right] \\
& -2 A_{1}\left(\sum_{r, s} \frac{1}{\omega_{r s}}+S_{2} z\right)+A_{1} S_{2} \delta .
\end{aligned}
$$

Note that the series (39a, b) are not absolutely convergent because the sum $S_{2}$ is conditionally convergent, which will be dealt with in Subsect. 4.5. The sum $\sum_{r, s}^{\prime} \frac{1}{\omega_{r s}}$ from (39a) and (40) is also not convergent, but since it doesn't appear in the derivative of $f_{\mathrm{m}}(z)$, it doesn't affect the heat flux and gradient fields. The relation (40) can be considered as a generalization of Filshtinsky's representation $[1,7]$ for a doubly-periodic array of sole fibers to the case of a doubly-periodic array of fiber pairs.

Because Weierstrass $\varsigma$ function is quasiperiodic and all its derivatives are doubly-periodic, it can be obtained that

$$
\begin{aligned}
& f_{\mathrm{m}}\left(z_{\mathrm{B}}\right)-f_{\mathrm{m}}\left(z_{\mathrm{A}}\right)=A_{0} \omega_{2}+2 A_{1}\left[2 \zeta\left(\frac{\omega_{2}}{2}\right)-S_{2} \omega_{2}\right], \\
& f_{\mathrm{m}}\left(z_{\mathrm{C}}\right)-f_{\mathrm{m}}\left(z_{\mathrm{B}}\right)=A_{0}\left(-\omega_{1}\right)+2 A_{1}\left[-2 \zeta\left(\frac{\omega_{1}}{2}\right)+S_{2} \omega_{1}\right] .
\end{aligned}
$$

As we can see, the right sides of the two Eqs. (41a, b) are constants, so it is proved that constructed complex potential $f_{\mathrm{m}}(z)$ expressed by (4) and (12) is quasi-periodic. By substituting (41) into (37) and then into (36), one obtains that

$$
\left\langle q_{1}\right\rangle+i\left\langle q_{2}\right\rangle=k_{\mathrm{m}} \bar{A}_{0}-2 k_{\mathrm{m}}\left(\pi A_{1}+\bar{S}_{2} \bar{A}_{1}-\bar{\varepsilon} \bar{A}_{1}\right)
$$

where

$$
\varepsilon=\varepsilon\left(\omega_{1}, \omega_{2}\right)=\frac{1}{\mathrm{i}}\left[\varsigma\left(\frac{\omega_{2}}{2}\right) \bar{\omega}_{1}-\varsigma\left(\frac{\omega_{1}}{2}\right) \bar{\omega}_{2}\right] .
$$

It can be seen from (42) that the average flux is only related to $A_{0}\left(=H_{1}^{0}+\mathrm{i} H_{2}^{0}\right)$ and the first coefficient $A_{1}$. The values of $\varepsilon$ for some typical arrays are listed in Yan et al. [50]. Note that the value of the conditionally convergent sum $S_{2}$ is dependent to the summation sequence. Therefore, for given external gradient $H_{j}^{0}$, the average flux and average gradient calculated from the potential (40) are dependent to the summation sequence of $S_{2}$. There exists a value of $S_{2}$ in the constructed potential (40), which makes the average gradient meet the boundary condition, i.e., the supplementary equation (30).

\subsection{Complete coefficient equations}

By substituting (34) and (42) into the supplementary equation (30), it is derived that the value of $S_{2}$ which makes the average gradient meet the boundary condition, satisfies:

$$
S_{2}=\pi \frac{\bar{A}_{1}}{A_{1}}+\varepsilon
$$

By substituting (44) into the coefficient equations (29), we obtain the complete coefficient equations without $S_{2}$ and $E_{2}(\delta)$ :

$$
\left\{\begin{array}{l}
\bar{A}_{1}=\frac{\lambda \eta_{1}}{2 \pi\left(1+\lambda \eta_{1}\right)}\left\{A_{0}-A_{1}[2 \varepsilon+\wp(\delta)]+\sum_{j=2}^{\infty} A_{j} \mathrm{C}_{j}^{1}\left[(-1)^{j} S_{j+1}-E_{j+1}(\delta)\right]\right\} \\
\bar{A}_{n}=\eta_{n}\left(\frac{\lambda}{2 \pi}\right)^{n} \sum_{j=1}^{\infty} A_{j} \mathrm{C}_{j+n-1}^{n}\left[(-1)^{j} S_{j+n}-E_{j+n}(\delta)\right], \quad n \geq 2
\end{array}\right.
$$

where $\lambda\left(=2 \pi R^{2}\right)$ is the fiber volume fraction for $V=1$. The coefficient equations (45) can be expressed in a vector and matrix form, just as it is detailed in Yan et al. [50]. By applying a square truncation to order $N$, the first $N$ expansion coefficients in the potential $f_{\mathrm{m}}(z)$ are obtained

It is worth noting that when $\delta$ is equal to the half of any of the periods, the considered case of a doubly-periodic array of fiber pairs is reduced into the case of a doubly-periodic array of sole fibers with the fundamental periods halved. 


\section{Effective conductivities}

After the expansion coefficients are determined through the coefficient equations (45), the average flux and average gradient can be calculated by (34) and (42). And then the effective conductivities $\left\langle k_{j k}\right\rangle$ are calculated by using the average field method:

$$
\left\langle q_{j}\right\rangle=-\left\langle k_{j k}\right\rangle\left\langle H_{k}\right\rangle, \quad j, k=1,2 .
$$

Detailed derivation of the formulae of the effective conductivities is given similarly in the previous paper [50], thus will not be repeated here.

When an appropriate truncation to an enough high order for (45) is applied, high-order numerical results of desired accuracy are obtained. When a truncation to finite order $N$ is applied, approximate analytical formulae of the effective conductivities of order $N$ are obtained, which are given with a moderate length for different fiber-pair arrays in the following.

\subsection{General doubly-periodic array of fiber pairs}

For the case of a general doubly-periodic array of fiber pairs as shown in Fig. 1, the effective conductivities are anisotropic in general. By a square truncation to order $N$, the approximate analytical formula in complex form is obtained as:

$$
\left\langle k_{11}\right\rangle+\mathrm{i}\left\langle k_{12}\right\rangle=k_{\mathrm{m}} \frac{a_{N}}{b_{N}},
$$

where the parameters $a_{N}$ and $b_{N}$ are from square truncation of the coefficient equation (45) for order $N$. When $N=1$,

$$
\begin{aligned}
& a_{1}=1-\left[1-\frac{1}{\pi}(2 \bar{\varepsilon}+\bar{\wp})+\frac{1}{4 \pi^{2}}|2 \varepsilon+\wp|^{2}\right] \eta_{1}^{2} \lambda^{2}, \\
& b_{1}=\left(1+\eta_{1} \lambda\right)^{2}-\frac{1}{4 \pi^{2}}|2 \varepsilon+\wp|^{2} \eta_{1}^{2} \lambda^{2} .
\end{aligned}
$$

When $N=2$,

$$
\begin{aligned}
a_{2}= & \left.1-\left[1-\frac{1}{\pi}(2 \bar{\varepsilon}+\varnothing)+\frac{1}{4 \pi^{2}}|2 \varepsilon+\wp|^{2}\right] \eta_{1}^{2} \lambda^{2}-\frac{1}{2 \pi^{3}}\left|E_{3}\right|^{2} \eta_{1} \eta_{2} \lambda^{3}-\frac{9}{16 \pi^{4}}\left|E_{4}-S_{4}\right|^{2}\right] \eta_{2}^{2} \lambda^{4} \\
& +\frac{1}{64 \pi^{6}}\left[\left|2 E_{3}^{2}-3(2 \varepsilon+\wp)\left(E_{4}-S_{4}\right)\right|^{2}+12 \pi\left(E_{4}-S_{4}\right)\left(2 \bar{E}_{3}^{2}-3(2 \bar{\varepsilon}+\wp)\left(\bar{E}_{4}-\bar{S}_{4}\right)\right)\right. \\
& \left.+36 \pi^{2}\left|E_{4}-S_{4}\right|^{2}\right] \eta_{1}^{2} \eta_{2}^{2} \lambda^{6}, \\
b_{2}= & \left(1+\eta_{1} \lambda\right)^{2}-\frac{1}{4 \pi^{2}}|2 \varepsilon+\wp|^{2} \eta_{1}^{2} \lambda^{2}-\frac{1}{2 \pi^{3}}\left|E_{3}\right|^{2} \eta_{1} \eta_{2} \lambda^{3}-\left[\frac{1}{2 \pi^{3}}\left|E_{3}\right|^{2} \eta_{1}^{2} \eta_{2}+\frac{9}{16 \pi^{4}}\left|E_{4}-S_{4}\right|^{2} \eta_{2}^{2}\right] \lambda^{4} \\
& -\frac{9}{8 \pi^{4}}\left|E_{4}-S_{4}\right|^{2} \eta_{1} \eta_{2}^{2} \lambda^{5}+\frac{1}{64 \pi^{6}}\left[\left|2 E_{3}^{2}-3(2 \varepsilon+\wp)\left(E_{4}-S_{4}\right)\right|^{2}-36 \pi^{2}\left|E_{4}-S_{4}\right|^{2}\right] \eta_{1}^{2} \eta_{2}^{2} \lambda^{6},
\end{aligned}
$$

where $\wp$ and $E_{n}(n=3,4,5, \ldots)$ denote $\wp(\delta)$ and $E_{n}(\delta)$ for short, respectively. Among all the parameters in the formulae (48) and (49), $\eta_{n}(n=1,2,3, \ldots)$ are the material parameters, $\lambda$ is the total fiber volume fraction, $\varepsilon, S_{n}, \wp$, and $E_{n}$ are the geometric parameters. Among the four geometric parameters, $\varepsilon$ and $S_{n}$ are related to the doubly-periodicity of fiber distribution, while $\wp$ and $E_{n}$ are additionally related to the fiber clustering. $\varepsilon, S_{n}, \wp$, and $E_{n}$ are complex numbers for general doubly-periodic arrays and generally-oriented fiber pairs. The calculations of $E_{n}$ are discussed in Sect. 3 .

The four geometric parameters are the functions of the fundamental complex periods $\left(\omega_{1}\right.$ and $\left.\omega_{2}\right)$ and the complex translation $\delta$, and then $a_{N}$ and $b_{N}$ in (47) are the functions of $\omega_{1}, \omega_{2}$, and $\delta$. When an doubly-periodic array is rotated by an angle of 90 degrees, that is, $\omega_{1}, \omega_{2}$, and $\delta$ are replaced by $\mathrm{i} \omega_{1}, \mathrm{i} \omega_{2}$, and $\mathrm{i} \delta$, the directions of the effective conductivities are rotated by an angle of 90 degrees, correspondingly. Therefore, it can be derived as

$$
\left\{\begin{array}{l}
\left\langle k_{11}\right\rangle+\mathrm{i}\left\langle k_{12}\right\rangle=k_{\mathrm{m}} \frac{a_{N}\left(\omega_{1}, \omega_{2}, \delta\right)}{b_{N}\left(\omega_{1}, \omega_{2}, \delta\right)} \\
\left\langle k_{22}\right\rangle-\mathrm{i}\left\langle k_{12}\right\rangle=k_{\mathrm{m}} \frac{a_{N}\left(\mathrm{i} \omega_{1}, \mathrm{i} \omega_{2}, \mathrm{i} \delta\right)}{b_{N}\left(\mathrm{i} \omega_{1}, \mathrm{i} \omega_{2}, \mathrm{i} \delta\right)}
\end{array} .\right.
$$




\subsection{Orthotropic doubly-periodic array of fiber pairs}

For orthotropic fiber arrays symmetric about $x_{1}$ axis or about $x_{2}$ axis, such as the rectangular or rhombic array of fiber pairs as shown in Fig. 2a, b, we have

$$
\begin{aligned}
& \bar{\varepsilon}=\varepsilon, \\
& \bar{S}_{2 m}=S_{2 m}, \quad S_{2 m-1}=0, \quad \text { for } \quad m \geq 2, \\
& \overline{\wp(\delta)}=\wp(\delta), \\
& \overline{E_{2 m}(\delta)}=E_{2 m}(\delta), \quad \text { for } m \geq 2, \\
& \overline{E_{2 m-1}(\delta)}=\left\{\begin{array}{cc}
E_{2 m-1}(\delta), & \text { arrays symmetric about } x_{1} \text { axis } \\
-E_{2 m-1}(\delta), & \text { arrays symmetric about } x_{2} \text { axis, }
\end{array} \text { for } m \geq 2 .\right.
\end{aligned}
$$

Therefore, it can be concluded that for such orthotropic fiber arrays, the geometric parameters $\varepsilon, S_{2 m}, \wp(\delta)$, and $E_{2 m}(\delta)$ are real. $E_{2 m-1}(\delta)$ are real for arrays symmetric about $x_{1}$ axis, while are pure imaginary for arrays symmetric about $x_{2}$ axis.
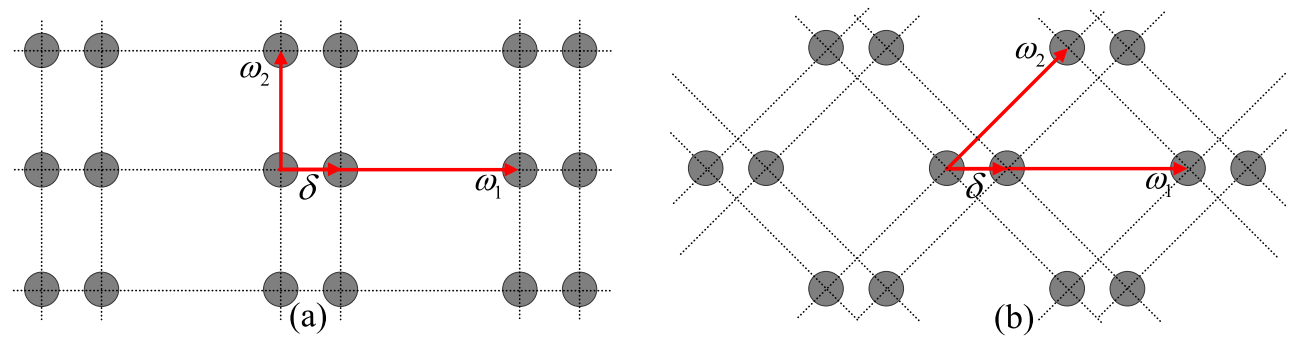

Fig. 2 Two kinds of orthotropic doubly-periodic array of fiber pairs: (a) Rectangular array of fiber pairs;

(b) Rhombic array of fiber pairs. The complex period $\omega_{1}$ is parallel to the $x_{1}$ axis.

For fiber arrays symmetric about $x_{1}$ axis, all the geometric parameters are real, and the coefficient equation (45) can be simplified [50]. As a result, the approximate analytical formula of the transverse effective conductivities can be written in the following form:

$$
\left\{\begin{array}{l}
\left\langle k_{11}\right\rangle=\left\langle k_{11}\left(\varepsilon, S_{4}, S_{6}, \wp, \eta_{n}\right)\right\rangle=k_{\mathrm{m}} \frac{c_{N}\left(1-\eta_{1} \lambda+\frac{2 \varepsilon+\wp}{2 \pi} \eta_{1} \lambda\right)+d_{N} \eta_{1} \lambda^{3}}{c_{N}\left(1+\eta_{1} \lambda+\frac{2 \varepsilon+\wp}{2 \pi} \eta_{1} \lambda\right)+d_{N} \eta_{1} \lambda^{3}} \\
\left\langle k_{22}\right\rangle=\frac{k_{\mathrm{m}}^{2}}{\left\langle k_{11}\left(\varepsilon, S_{4}, S_{6}, \wp,-\eta_{n}\right)\right\rangle} \\
\left\langle k_{12}\right\rangle=0,
\end{array}\right.
$$

where the parameters $c_{N}$ and $d_{N}$ are from square truncation of the coefficient equation (45) for order $N$. It can be seen from (56) that the effective conductivities in two principle directions satisfy the reciprocal relation [23,36], which also holds for orthotropic arrays of sole fibers [50].

When $N=1$, it is obtained that $c_{1}=1, d_{1}=0$. Then the obtained first order approximate analytical formula is

$$
\left\langle k_{11}\right\rangle=k_{\mathrm{m}} \frac{1-\eta_{1} \lambda+\frac{2 \varepsilon+\wp}{2 \pi} \eta_{1} \lambda}{1+\eta_{1} \lambda+\frac{2 \varepsilon+\wp}{2 \pi} \eta_{1} \lambda},
$$

which can be seen as a further modification of the first order approximate analytical formula [50] for orthotropic array of sole fibers, by introducing the parameter $\wp$ to consider the influence of the fiber clustering. The latter is a modification of the classical Maxwell-Garnett estimation [29], by introducing the parameter $\varepsilon$ to consider the influence of the orthotropic fiber distribution. When $N=2$,

$$
c_{2}=1+\frac{3}{4 \pi^{2}}\left(E_{4}-S_{4}\right) \eta_{2} \lambda^{2}, \quad d_{2}=-\frac{1}{4 \pi^{3}} E_{3}^{2} \eta_{2} .
$$

When $N=3$,

$$
c_{3}=c_{2}+\frac{5}{4 \pi^{3}}\left(E_{6}+S_{6}\right) \eta_{3} \lambda^{3}-\frac{3}{16 \pi^{5}}\left(4 E_{5}^{2}-5 E_{4} E_{6}+5 E_{6} S_{4}-5 E_{4} S_{6}+5 S_{4} S_{6}\right) \eta_{2} \eta_{3} \lambda^{5},
$$




$$
\begin{aligned}
d_{3}= & d_{2}-\frac{3}{16 \pi^{4}}\left(E_{4}+S_{4}\right)^{2} \eta_{3} \lambda \\
& -\frac{1}{64 \pi^{6}}\left[9 E_{4}^{3}+9 E_{4}^{2} S_{4}-24 E_{3} E_{5} S_{4}-9 S_{4}^{3}-3 E_{4}\left(8 E_{3} E_{5}+3 S_{4}^{2}\right)+20 E_{3}^{2}\left(E_{6}+S_{6}\right)\right] \eta_{2} \eta_{3} \lambda^{3} .
\end{aligned}
$$

In contrast, for fiber arrays symmetric about $x_{2}$ axis, $E_{2 m-1}(\delta)$ are pure imaginary, the simplification of the coefficient equation (45) provided similarly in Yan et al. [50] is not applicable. However, the arrays symmetric about $x_{2}$ axis can always be rotated to be symmetric about $x_{1}$ axis, by an angle of 90 degrees. Corresponding to the rotation, the geometric parameters $\varepsilon, S_{2 m}, \wp(\delta), E_{2 m}(\delta)$, and $E_{2 m-1}(\delta)$ in Eqs. (56)-(59) should be replaced by $-\varepsilon,(-1)^{m} S_{2 m},-\wp(\delta),(-1)^{m} E_{2 m}(\delta)$, and $\mathrm{i}(-1)^{m-1} E_{2 m-1}(\delta)$, respectively.

\subsection{Triangular array of fibers}

As shown in Fig. 3c, a triangular array of sole fibers can be seen as a hexagonal array of special fiber pairs. The two fundamental periods and the complex translation satisfy $\omega_{2}=\mathrm{e}^{\mathrm{i} \pi / 3} \omega_{1}$ and $\delta=\left(\omega_{1}+\omega_{2}\right) / 3$. Such a special array is symmetric about $x_{2}$ axis and rotationally symmetric by 120 degrees. We have $\varepsilon=\left(\mathrm{e}^{\mathrm{i} 2 \pi / 3}\right)^{2} \varepsilon, S_{2 m}=\left(\mathrm{e}^{\mathrm{i} 2 \pi / 3}\right)^{2 m} S_{2 m}$, $\wp(\delta)=\left(\mathrm{e}^{\mathrm{i} 2 \pi / 3}\right)^{2} \wp(\delta)$, and $E_{m}(\delta)=\left(\mathrm{e}^{\mathrm{i} 2 \pi / 3}\right)^{m} E_{m}(\delta)$, thus $\varepsilon=0, \wp(\delta)=0, S_{2 m}=0$ for $2 m \neq 6 l(m \geq 2, l \geq 1)$ and $E_{m}(\delta)=0$ for $m \neq 3 l(m \geq 3, l \geq 1)$. Additionally, from (24) and (27), we have $S_{6}=-\frac{1}{35} E_{3}^{2}$. Considering these properties of the geometric parameters, the approximate analytical formulae can be written as

$$
\left\langle k_{12}\right\rangle=0, \quad\left\langle k_{11}\right\rangle=\left\langle k_{22}\right\rangle=k_{\mathrm{m}} \frac{c_{N}\left(1-\eta_{1} \lambda\right)+d_{N} \eta_{1} \lambda^{3}}{c_{N}\left(1+\eta_{1} \lambda\right)+d_{N} \eta_{1} \lambda^{3}},
$$

It can be seen that the transverse conductivity is isotropic, which agrees with the prediction from the rotational symmetry of the triangular array. When the order $N=1$, it can be obtained that $c_{1}=1, d_{1}=0$. The obtained first order approximate analytical formula is exactly the classical Maxwell-Garnett estimation [29]:

$$
\left\langle k_{11}\right\rangle=\left\langle k_{22}\right\rangle=k_{\mathrm{m}} \frac{1-\eta_{1} \lambda}{1+\eta_{1} \lambda} .
$$

For order $N=4$,

$$
c_{4}=1-\frac{225}{1568 \pi^{6}} E_{3}^{4} \eta_{2} \eta_{4} \lambda^{6}, \quad d_{4}=\frac{1}{4 \pi^{3}} E_{3}^{2} \eta_{2},
$$

For order $N=8$,

$$
\begin{aligned}
c_{8}= & c_{4}+\frac{1}{224 \pi^{9}} E_{3}^{6}\left(35 \eta_{4} \eta_{5}+2 \eta_{2} \eta_{7}\right) \lambda^{9}-\frac{225}{831014912 \pi^{12}} E_{3}^{8}\left(373030 \eta_{5} \eta_{7}+131769 \eta_{4} \eta_{8}\right) \lambda^{12} \\
& +\frac{9801}{87808 \pi^{15}} E_{3}^{10} \eta_{7} \eta_{8} \lambda^{15}+\frac{641731205}{93073670144 \pi^{18}} E_{3}^{12} \eta_{2} \eta_{4} \eta_{5} \eta_{7} \lambda^{18} \\
& -\frac{1099917225}{93073670144 \pi^{21}} E_{3}^{14} \eta_{2} \eta_{4} \eta_{7} \eta_{8} \lambda^{21}+\frac{255957890096205}{49327555997597696 \pi^{24}} E_{3}^{16} \eta_{4} \eta_{5} \eta_{7} \eta_{8} \lambda^{24}, \\
d_{8}= & d_{4}-\frac{169}{15680 \pi^{6}} E_{3}^{4} \eta_{5} \lambda^{3}+\frac{1}{3136 \pi^{9}} E_{3}^{6} \eta_{8} \lambda^{6}+\frac{123245}{4917248 \pi^{12}} E_{3}^{8} \eta_{2} \eta_{4} \eta_{5} \lambda^{9} \\
& -\frac{1}{16620298240 \pi^{15}} E_{3}^{10} \eta_{2}\left(369442766 \eta_{5} \eta_{7}+127765125 \eta_{4} \eta_{8}\right) \lambda^{12} \\
& +\frac{1}{14520489728 \pi^{18}} E_{3}^{12}\left(2429045 \eta_{4} \eta_{5}+421654016 \eta_{2} \eta_{7}\right) \eta_{8} \lambda^{15} \\
& +\frac{783272169}{930736701440 \pi^{21}} E_{3}^{14} \eta_{5} \eta_{7} \eta_{8} \lambda^{18}+\frac{135586583950205}{197310223990390784 \pi^{27}} E_{3}^{18} \eta_{2} \eta_{4} \eta_{5} \eta_{7} \eta_{8} \lambda^{24},
\end{aligned}
$$

where $E_{3}=-11.5449$ i. It can be seen that, $c_{N}$ and $d_{N}$ contain only the terms $\lambda^{l}$ which have $l$ divisible by three.

Interestingly, comparing the present approximate analytical formula (60) for triangular array (Fig. 3c) with those [50] for square array (Fig. 3b) and hexagonal array (Fig. 3a), we can see that they have similar forms. The difference among them reflects different symmetric characteristics of the three special symmetric arrays. 


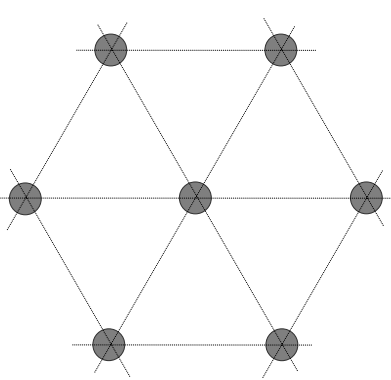

(a)

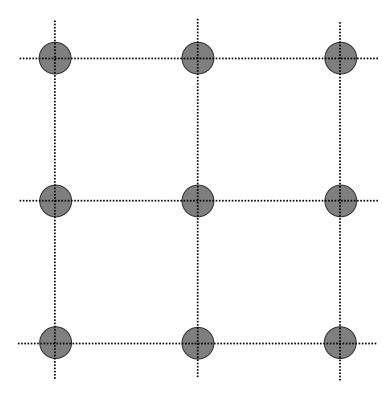

(b)

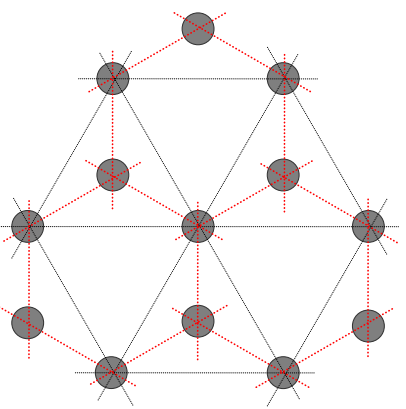

(c)

Fig. 3 Three special symmetric fiber arrays: (a) Hexagonal array; (b) Square array; (c) Triangular array.

\section{Comparisons of results and discussions}

\subsection{Comparisons with the known formulae}

As mentioned in the introduction, Rylko [43] applied the method of functional equations to calculate the conductivity of a rectangular array of cylinder pairs and obtained approximate analytical formulae of effective conductivities of perfectly conducting cylinders. In order to compare the present formulae with the known formulae (Eqs. (25), (26) in [43]), the conjugation of the approximate analytical formula (47) is expanded into Taylor series

$$
\begin{aligned}
\frac{\left\langle k_{11}\right\rangle-\mathrm{i}\left\langle k_{12}\right\rangle}{k_{\mathrm{m}}}= & 1-2 \eta_{1} \lambda+\frac{1}{\pi}(2 \varepsilon+\wp) \eta_{1}^{2} \lambda^{2} \\
& +\frac{1}{2 \pi^{2}}\left[2 \pi(2 \bar{\varepsilon}+\varnothing-2 \varepsilon-\wp)-|2 \varepsilon+\wp|^{2}\right] \eta_{1}^{3} \lambda^{3}+O\left(\lambda^{4}\right) .
\end{aligned}
$$

This formula is for a general doubly-periodic array of generally-oriented fiber pairs.

For a rectangular array of generally-oriented fiber pairs, $\bar{\varepsilon}=\varepsilon$, then Eq. (64) is rewritten as

$$
\frac{\left\langle k_{11}\right\rangle-\mathrm{i}\left\langle k_{12}\right\rangle}{k_{\mathrm{m}}}=1-2 \eta_{1} \lambda+\frac{1}{\pi}(2 \varepsilon+\wp) \eta_{1}^{2} \lambda^{2}+\frac{1}{2 \pi^{2}}\left[2 \pi(\bar{\wp}-\wp)-|2 \varepsilon+\wp|^{2}\right] \eta_{1}^{3} \lambda^{3}+O\left(\lambda^{4}\right) .
$$

For a rectangular array of fiber pairs symmetric about $x_{1}$ axis, $\bar{\varnothing}=\wp$ in addition, then Eq. (65) becomes

$$
\frac{\left\langle k_{11}\right\rangle-\mathrm{i}\left\langle k_{12}\right\rangle}{k_{\mathrm{m}}}=1-2 \eta_{1} \lambda+\frac{1}{\pi}(2 \varepsilon+\wp) \eta_{1}^{2} \lambda^{2}-\frac{1}{2 \pi^{2}}(2 \varepsilon+\wp)^{2} \eta_{1}^{3} \lambda^{3}+O\left(\lambda^{4}\right),
$$

where for a square array, $\varepsilon=0$ in addition.

Note that $\eta_{1}=-1$ for perfectly conducting cylinders (fibers). Considering the relations of the different parameters and comparing Eqs. (65), (66) with the Eqs. (25), (26) in [43], it can be concluded that, for the case of rectangular array of fiber pairs symmetric about $x_{1}$ axis, the present approximate analytical formula coincides with the formula (25) in [43].

\subsection{Validity and accuracy}

In order to verify the validity and accuracy of the present solutions, variations of effective conductivities with the truncation order $N$ for different fiber arrays are listed in Table 1. Perfect fiber-matrix interface is considered. The fiber-matrix conductivity ratio $k_{\mathrm{f}} k_{\mathrm{m}}=50$ or 120 for the convenience of comparing with available results in the references $[17,21,36,51]$. For different fiber arrays, the limiting volume fractions, $\lambda_{\lim }$, corresponding to the case of fibers touching with each other, are also given in the table. With the volume fraction, $\lambda$, approaching the limiting value, the truncation order $N$ required to reach a given accuracy increases. In Table 1 , volume fractions are chosen to obtain convergent results to the accuracy shown in the table within $N=20$. It can be seen that such volume fractions are very close to the limiting values. The underlined values in Table 1 are calculated from (49), (59), and (63), from which high accuracy of the approximate analytical formulae can be observed.

Note that a doubly-periodic array of sole fibers can be seen as a special case of a doubly-periodic array of fiber pairs with one of the fundamental periods doubled, so the present solution is also applicable for a doubly-periodic array of sole fibers, such as the hexagonal array, square array and rhombic array. Perfect agreement can be seen with the results by Perrins et al. [36] and Jiang et al. [21], as well as the results by using EEVM [51] and AHM [17]. 


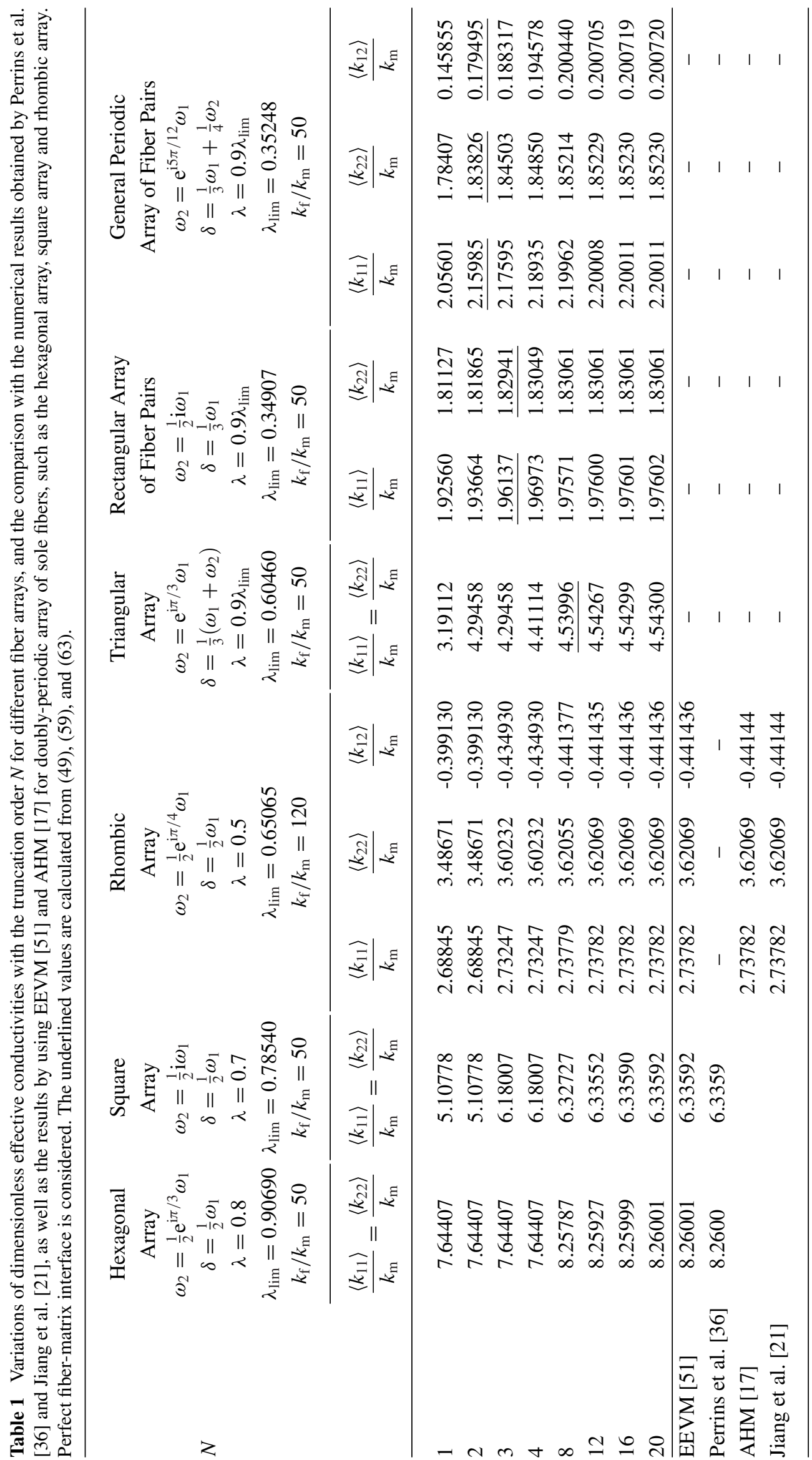




\subsection{Influence of the fiber pair}

A fiber pair can be seen as a fiber cluster containing two fibers. The influence of fiber/particle clustering is investigated in existing works $[10,30,44]$ by using random microstructure model. In the present study using periodic model, the fiber clusters, i.e. fiber pairs, are in a fixed doubly-periodic array, in order to investigate the influence of pairwise interaction in fiber pairs, separately.

Two kinds of orthotropic doubly-periodic fiber-pair arrays: rectangular array of fiber pairs (Fig. 2a) and rhombic array of fiber pairs (Fig. 2b), are taken for example. As shown in Fig. 2, $\omega_{1}$ and $\delta$ are real, $\omega_{2}=\frac{i}{2} \omega_{1}$ for the rectangular array in Fig. 2a and $\omega_{2}=\frac{\sqrt{2}}{2} \mathrm{e}^{\mathrm{i} \frac{\pi}{4}} \omega_{1}$ for the rhombic array in Fig. 2b. Initially, $\delta=\frac{1}{2} \omega_{1}$, all the fibers are in a square array (Fig. 2a) and in a rotated square array (Fig. 2b), so the effective conductivities in two orthotropic directions are the same. With the two fibers in fiber pairs approaching, variations of dimensionless effective conductivities are depicted in Fig. 4a and $\mathrm{b}$ at different fiber volume fraction, for the two fiber-pair arrays, respectively. The fiber-matrix conductivity ratio $k_{\mathrm{f}} / k_{\mathrm{m}}=$ 50. The distance of the pair of fibers is equal to $\delta$, whose minimum value is $2 R$ when the pair of fibers comes to contact. Therefore, $\frac{1}{2} \leq 1-\frac{\delta}{\omega_{1}} \leq 1-\sqrt{\frac{2 \lambda}{\pi}} \frac{1}{\omega_{1}}$, where $\omega_{1}$ is determined by (2). It can be seen from Fig. 4 that for both of the two fiber-pair arrays the effective conductivity along the fiber-pair orientation increases obviously, and it is more obvious as the fiber volume fraction increases. In contrast, the effective conductivity perpendicular to the fiber-pair orientation remains almost constant (decreases a little bit). That is, clustering of high-conductivity fibers increases the overall conductivity of composites, especially when fiber volume fraction is high.

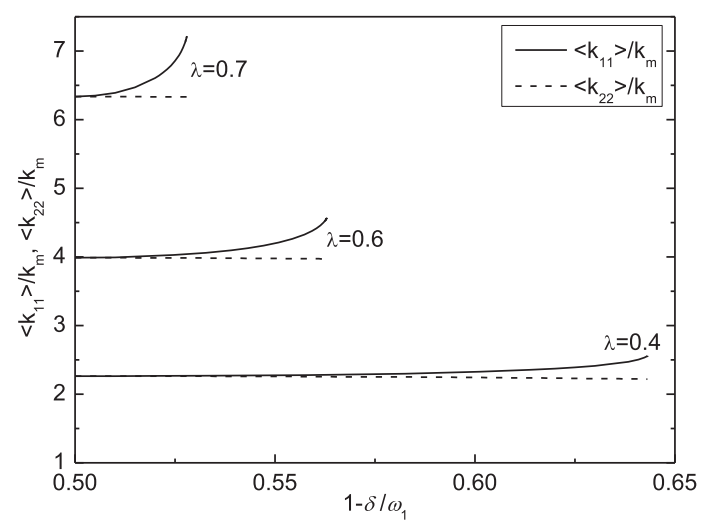

(a)

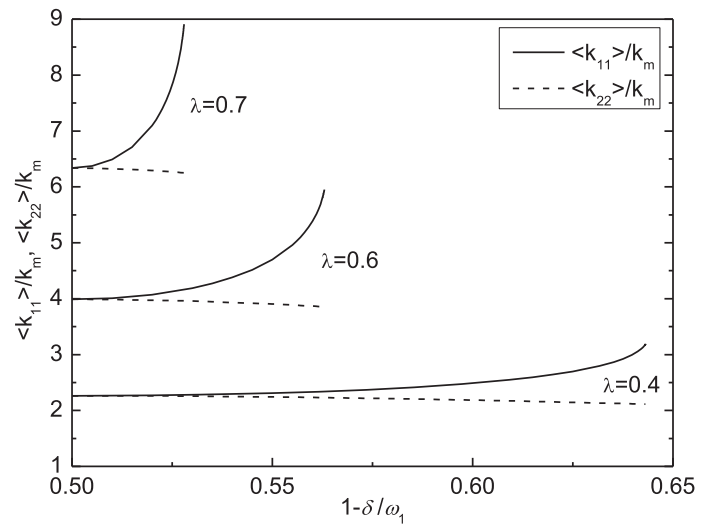

(b)

Fig. 4 Variations of dimensionless effectiveconductivities with two fibers in fiber pairs approachingfor different fibervolume fraction, for two kinds of orthotropic doubly-periodic fiber-pairarrays: (a) Rectangular array of fiber pairs (Fig. 2(a)); (b) Rhombic array of fiber pairs (Fig. 2(b)). $\delta$ is equal to the distance of the two fibers in fiber pairs. Fiber-matrix conductivity ratio $k_{f} / k_{m}=50$.

\subsection{Triangular fiber array}

Triangular fiber array (Fig. 3c) is another special symmetric array besides the square array (Fig. 3b) and hexagonal array (Fig. 3a) which results into transversely isotropic effective property. From hexagonal array to square array to triangular array, the fiber-distribution uniformity and limiting volume fraction $\lambda_{\lim }$ decrease. The limiting volume fractions for the three fiber arrays are listed in Table 1. In Fig. 5, variations of the dimensionless effective conductivities for the three fiber arrays with fiber volume fraction $\lambda$ are shown and compared with the experimental data [45] of fiber glass reinforced plastics $\left(k_{\mathrm{f}} / k_{\mathrm{m}}=4.4\right)$ and graphite fiber reinforced plastics $\left(k_{\mathrm{f}} / k_{\mathrm{m}}=666\right)$. The upper bound and lower bound are due to Hashin and Shtrikman [18]. It can be seen from Fig. 5 that the effective conductivities predicted for the three fiber arrays are within the two bounds. The effective conductivity increases rapidly with the volume fraction approaching the limiting value. The reason is that when high-conductivity fibers approach to contact with each other, they tend to produce a path of heat flow [9] and this would contribute to an increase in the effective conductivity. At a volume fraction within the limiting values, the effective conductivity for the triangular array is larger than that for the square array, and that for the square array larger than the hexagonal array. That is, the effective conductivity increases with the decrease of the fiber-distribution uniformity.

In Table 2, values of dimensionless effective conductivity for the triangular array are listed for various values of the fiber-matrix conductivity ratio $k_{\mathrm{f}} / k_{\mathrm{m}}$ and various fiber volume fractions $\lambda$. Table 3 gives the minimum truncation order $N$ 
Table 2 For the triangular array, values of dimensionless effective conductivity for various values of the fiber-matrix conductivity ratio $k_{\mathrm{f}} / k_{\mathrm{m}}$ and various fiber volume fractions $\lambda$. The limiting volume fraction of fiber is 0.60460 when fibers are in contact with each other.

\begin{tabular}{|c|c|c|c|c|c|c|c|c|}
\hline \multirow[b]{2}{*}{$\lambda$} & \multicolumn{8}{|c|}{$<k_{11}>/ k_{\mathrm{m}}=<k_{22}>/ k_{\mathrm{m}}$} \\
\hline & $k_{\mathrm{f}} / k_{\mathrm{m}}=2$ & $k_{\mathrm{f}} / k_{\mathrm{m}}=3.5$ & $k_{\mathrm{f}} / k_{\mathrm{m}}=5$ & $k_{\mathrm{f}} / k_{\mathrm{m}}=10$ & $k_{\mathrm{f}} / k_{\mathrm{m}}=20$ & $k_{\mathrm{f}} / k_{\mathrm{m}}=50$ & $k_{\mathrm{f}} / k_{\mathrm{m}}=100$ & $k_{\mathrm{f}} / k_{\mathrm{m}}=\infty$ \\
\hline 0.1 & 1.0690 & 1.1177 & 1.1429 & 1.1784 & 1.1991 & 1.2128 & 1.2176 & 1.2225 \\
\hline 0.2 & 1.1430 & 1.2507 & 1.3091 & 1.3940 & 1.4457 & 1.4804 & 1.4927 & 1.5054 \\
\hline 0.3 & 1.2230 & 1.4043 & 1.5082 & 1.6679 & 1.7704 & 1.8418 & 1.8676 & 1.8945 \\
\hline 0.4 & 1.3104 & 1.5877 & 1.7595 & 2.0460 & 2.2468 & 2.3957 & 2.4515 & 2.5108 \\
\hline 0.46 & 1.3674 & 1.7186 & 1.9504 & 2.3665 & 2.6838 & 2.9349 & 3.0327 & 3.1391 \\
\hline 0.5 & 1.4075 & 1.8181 & 2.1037 & 2.6517 & 3.1052 & 3.4898 & 3.6462 & 3.8210 \\
\hline 0.52 & 1.4283 & 1.8726 & 2.1910 & 2.8283 & 3.3852 & 3.8809 & 4.0892 & 4.3268 \\
\hline 0.54 & 1.4497 & 1.9308 & 2.2876 & 3.0382 & 3.7408 & 4.4085 & 4.7022 & 5.0474 \\
\hline 0.56 & 1.4718 & 1.9934 & 2.3959 & 3.2961 & 4.2192 & 5.1839 & 5.6393 & 6.2016 \\
\hline 0.58 & 1.4944 & 2.0615 & 2.5193 & 3.6296 & 4.9297 & 6.5217 & 7.3781 & 8.5488 \\
\hline 0.59 & 1.5060 & 2.0979 & 2.5885 & 3.8422 & 5.4605 & 7.7331 & 9.1213 & 11.2521 \\
\hline 0.6 & 1.5178 & 2.1362 & 2.6643 & 4.1074 & 6.2641 & 10.1902 & 13.4008 & 20.4111 \\
\hline 0.604 & 1.5225 & 2.1521 & 2.6969 & 4.2375 & 6.7657 & 12.6223 & 19.6251 & 57.2348 \\
\hline 0.6045 & 1.5231 & 2.1541 & 2.7011 & 4.2552 & 6.8453 & 13.1973 & 21.8799 & 140.8420 \\
\hline 0.60459 & 1.5233 & 2.1545 & 2.7019 & 4.2585 & 6.8602 & 13.3223 & 22.5019 & 450.5 \\
\hline
\end{tabular}

Table 3 For the triangular array, the minimum truncation order $N$ of the solution required to obtain effective conductivity to the accuracy quoted in Table 2.

N

$\lambda \quad k_{\mathrm{f}} / k_{\mathrm{m}}=2 \quad k_{\mathrm{f}} / k_{\mathrm{m}}=3.5 \quad k_{\mathrm{f}} / k_{\mathrm{m}}=5 \quad k_{\mathrm{f}} / k_{\mathrm{m}}=10 \quad k_{\mathrm{f}} / k_{\mathrm{m}}=20 \quad k_{\mathrm{f}} / k_{\mathrm{m}}=50 \quad k_{\mathrm{f}} / k_{\mathrm{m}}=100 \quad k_{\mathrm{f}} / k_{\mathrm{m}}=\infty$

\begin{tabular}{|c|c|c|c|c|c|c|c|c|}
\hline 0.1 & 1 & 2 & 2 & 2 & 2 & 2 & 2 & 2 \\
\hline 0.2 & 2 & 2 & 2 & 2 & 2 & 2 & 2 & 2 \\
\hline 0.3 & 2 & 2 & 5 & 5 & 5 & 5 & 5 & 5 \\
\hline 0.4 & 2 & 5 & 5 & 7 & 8 & 8 & 8 & 8 \\
\hline 0.46 & 5 & 7 & 8 & 8 & 10 & 10 & 10 & 10 \\
\hline 0.5 & 5 & 8 & 10 & 11 & 11 & 13 & 13 & 13 \\
\hline 0.52 & 5 & 9 & 10 & 13 & 13 & 14 & 14 & 14 \\
\hline 0.54 & 7 & 10 & 11 & 13 & 16 & 16 & 16 & 17 \\
\hline 0.56 & 8 & 11 & 13 & 16 & 19 & 20 & 20 & 22 \\
\hline 0.58 & 8 & 13 & 16 & 22 & 25 & 28 & 28 & 31 \\
\hline 0.59 & 8 & 14 & 19 & 25 & 31 & 37 & 37 & 40 \\
\hline 0.6 & 10 & 16 & 22 & 34 & 46 & 58 & 64 & 73 \\
\hline 0.604 & 10 & 16 & 25 & 43 & 73 & 121 & 151 & 211 \\
\hline 0.6045 & 10 & 16 & 25 & 46 & 85 & 175 & 265 & 523 \\
\hline 0.60459 & 10 & 16 & 25 & 46 & 88 & 202 & 367 & $1.5 \times 10^{3}$ \\
\hline
\end{tabular}




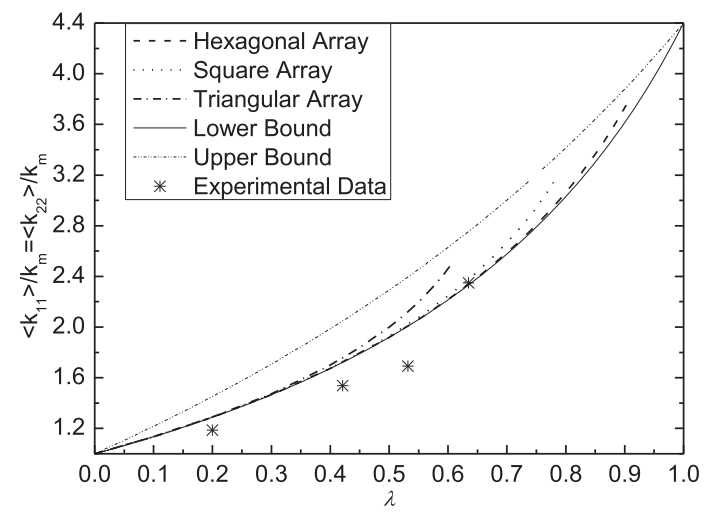

(a)

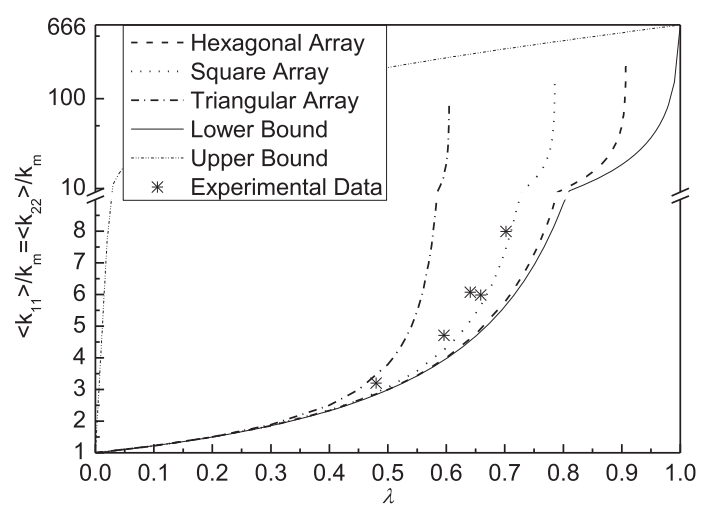

(b)

Fig. 5 The dimensionless effectiveconductivities are shown as a function of fiber volume fraction $\lambda$, for three kinds of special symmetricfiber arrays (Hexagonal array, square array and triangular array), and compared with the experimental data [45]: (a) fiber glass reinforced plastics $\left(k_{f} / k_{m}=4.4\right)$; (b) graphite fiber reinforced plastics $\left(k_{f} / k_{m}=666\right)$. The upper bound and lower bound are due toHashin and Shtrikman [18].

of the solution required to obtain effective conductivity to the accuracy quoted in Table 2 . It can be seen that the required truncation order $N$ increases with the volume fraction, $\lambda$, approaching the limiting value $\left(\lambda_{\lim }=0.60460\right)$ and with the increase of the fiber-matrix conductivity ratio $k_{\mathrm{f}} / k_{\mathrm{m}}$. Table 3 also gives a reference to determine the region of applicability of the approximate analytical formulae (60)-(63).

\section{Conclusions}

A complex variable method is proposed to calculate effective transport properties of composites with a doubly-periodic array of fiber pairs. By using the centrosymmetry of the problem, the method of Rayleigh and Natanzon-Filshtinsky's approach can be simply extended to the problems with two fibers per unit cell. The infinite system constructed in this paper only slightly complicates Rayleigh's system for the problems with one fiber per unit cell. Approximate analytical formulae of effective transport properties with moderate length for different fiber-pair arrays are obtained. In particular, an approximate analytical formula for the triangular fiber array is obtained, which is an extension of those for the square array and hexagonal array. Triangular fiber array is another special symmetric fiber array which results into transversely isotropic effective property, thus is of interest in engineering application.

Numerical results reveal that clustering of high-conductivity fibers increases the overall conductivity of composites, especially when fiber volume fraction is high. The comparison of the results for the three special symmetric fiber arrays reveals that the triangular fiber array has the highest conductivity. The effective conductivity increases with the decrease of the fiber-distribution uniformity. In addition, high accuracy and validity of the present approximate analytical formulae is verified in the numerical examples.

Though the solutions are derived and discussed for the case of perfect fiber-matrix interface, they can be easily extended and applied to the cases of contact resistance interface and coating interface between fiber and matrix.

Acknowledgements The authors would like to thank the reviewers for the helpful and constructive comments. The work is supported by the National Natural Science Foundation of China under Grant NNSFC 11302009, Aviation Science Foundation of China (2013ZBN3004), the Fundamental Research Funds for the Central Universities and The Open Fund of LNM.

\section{References}

[1] I. Andrianov and V. Mityushev, Exact and "exact" formulae in the theory of composites, arXiv:1708.02137v1 (2017).

[2] I. Aviram and Y. Yeivin, Neutron transport in a doubly-periodic array of absorbing multipoles, J. Nucl. Energy 26(2), 61-74 (1972).

[3] L. P. Castro, D. Kapanadze, and E. Pesetskaya, A heat conduction problem of 2D unbounded composites with imperfect contact conditions, ZAMM - Z. Angew. Math. Mech. 95(9), 952-965 (2015).

[4] J. E. Drummond and M. I. Tahir, Laminar viscous flow through regular arrays of parallel solid cylinders, Int. J. Multiph. Flow 10(5), 515-540 (1984). 
[5] P. Drygas and V. Mityushev, Effective conductivity of unidirectional cylinders with interfacial resistance, Q. J. Mech. Appl. Math. 62(3), 235-262 (2009).

[6] L. A. Fil'shtinskii, Stresses and displacements in an elastic sheet weakened by a doubly-periodic set of equal circular holes, J. Appl. Math. Mech. 28(3), 530-543 (1964).

[7] L. Filshtinsky and V. Mityushev, Mathematical Models of Elastic and Piezoelectric Fields in Two-Dimensional Composites (Springer, New York, 2014).

[8] R. Gajić, D. Jovanović, K. Hingerl, R. Meisels, and F. Kuchar, 2D photonic crystals on the Archimedean lattices (tribute to Johannes Kepler (1571-1630)), Opt. Mater. 30(7), 1065-1069 (2008).

[9] D. Ganapathy, K. Singh, P. E. Phelan, and R. Prasher, An effective unit cell approach to compute the thermal conductivity of composites with cylindrical particles, J. Heat Transf. 127(6), 553-559 (2005).

[10] S. Ghosh, Z. Nowak, and K. Lee, Quantitative characterization and modeling of composite microstructures by Voronoi cells, Acta. Mater. 45(6), 2215-2234 (1997).

[11] S. Gluzman, V. Mityushev, and W. Nawalaniec, Cross-properties of the effective conductivity of the regular array of ideal conductors, Arch. Mech. 66(4), 287-301 (2014).

[12] S. Gluzman and V. Mityushev, Series, index and threshold for random 2D composite, Archive of Mechanics 67(1), 75-93 (2015).

[13] S. Gluzman, V. Mityushev, W. Nawalaniec, and G. Starushenko: Effective Conductivity and Critical Properties of a Hexagonal Array of Superconducting Cylinders. In: Pardalos, P.M. and Rassias, T.M. (eds.) Contributions in Mathematics and Engineering. In Honor of Constantin Caratheodory (Springer International Publishing, 2016).

[14] S. Gluzman, V. Mityushev, W. Nawalaniec, and G. Sokal, Random composite: stirred or shaken?, Arch. Mech. 68(3), 1-13 (2016).

[15] Y. A. Godin, The effective conductivity of a periodic lattice of circular inclusions, J. Math. Phys. 53(6), 063703 (2012).

[16] R. Guinovart-Díaz, P. Yan, R. Rodríguez-Ramos, J. C. López-Realpozo, C. P. Jiang, J. Bravo-Castillero, and F. J. Sabina, Effective properties of piezoelectric composites with parallelogram periodic cells, Int. J. Eng. Sci. 53(4), 58-66 (2012).

[17] R. Guinovart-Diaz, J. C. Lopez-Realpozo, R. Rodriguez-Ramos, J. Bravo-Castillero, M. Ramirez, H. Camacho-Montes, and F. J. Sabina, Influence of parallelogram cells in the axial behaviour of fibrous composite, Int. J. Eng. Sci. 49(1), 75-84 (2011).

[18] Z. Hashin and S. Shtrikman, A variational approach to the theory of the effective magnetic permeability of multiphase materials, J. Appl. Phys. 33(10), 3125-3131 (1962).

[19] J. Huang, Integral representations of harmonic lattice sums, J. Math. Phys. 40(10), 5240-5246 (1999).

[20] F. P. Incropera, D. P. Dewitt, T. L. Bergman, and A. S. Lavine, Fundamentals of Heat and Mass Transfer, 6th ed. (John Wiley \& Sons, New York, 2007).

[21] C. P. Jiang, Y. L. Xu, Y. K. Cheung, and S. H. Lo, A rigorous analytical method for doubly periodic cylindrical inclusions under longitudinal shear and its application, Mech. Mater. 36(3), 225-237 (2004).

[22] F. H. Kaatz, A. Bultheel, and T. Egami, Order in mathematically ideal porous arrays: the regular tilings, http://nalag.cs .kuleuven.be/papers/ade/regulartiles/index.html (2010).

[23] J. B. Keller, A theorem on the conductivity of a composite medium, J. Math. Phys. 5(4), 548-549 (1964).

[24] V. I. Kushch, I. Sevostianov, and V. S. Chernobai, Effective conductivity of composite with imperfect contact between elliptic fibers and matrix: Maxwell's homogenization scheme, Int. J. Eng. Sci. 83, 146-161 (2014).

[25] S. Lang, Elliptic Functions. 2nd edition (Springer, New York, 1987).

[26] J. C. Lopez-Realpozo, R. Rodriguez-Ramos, R. Guinovart-Diaz, J. Bravo-Castillero, and F. J. Sabina, Transport properties in fibrous elastic rhombic composite with imperfect contact condition, Int. J. Mech. Sci. 53(2), 98-107 (2011).

[27] R. C. McPhedran, Transport properties of cylinder pairs and of the square array of cylinders, Proc. R. Soc. Lond. A 408(1834), 31-43 (1986).

[28] R. C. McPhedran, L. Poladian, and G. W. Milton, Asymptotic studies of closely spaced, highly conducting cylinders, Proc. R. Soc. Lond. A 196(415), 185-196 (1988).

[29] G. W. Milton, The Theory of Composites (Cambridge University Press, Cambridge, 2002).

[30] L. Mishnaevsky Jr, Computational Mesomechanics of Composites: Numerical analysis of the effect of microstructures of composites on their strength and damage resistance (John Wiley \& Sons, Singapore, 2007).

[31] V. Mityushev, Representative cell in mechanics of composites and generalized Eisenstein-Rayleigh sums, Complex Var. Elliptic Equ. 51(8-11), 1033-1045 (2006).

[32] V. Mityushev, E. Pesetskaya, and S. Rogosin, Analytical Methods for Heat Conduction in Composites and Porous Media. In: Öchsner, A., Murch, G.E., and Lemos, M.J.S.d. (eds.) Cellular and Porous Materials: Thermal Properties Simulation and Prediction, pp. 67-78 (Wiley-VCH Verlag GmbH \& Co. KGaA, Weinheim, 2008).

[33] V. Mityushev and P. M. Adler, Longitudinal permeability of spatially periodic rectangular arrays of circular cylinders I. A single cylinder in the unit cell, ZAMM - Z. Angew. Math. Mech. 82(5), 335-345 (2002).

[34] V. Mityushev, Random 2D composites and the generalized method of Schwarz, Adv. Math. Phys. 2015(6), 1-15 (2015).

[35] S. Nemat-Nasser and M. Hori, Micromechanics: Overall Properties of Heterogeneous Materials (Elsevier, Amsterdam, 1999).

[36] W. T. Perrins, D. R. McKenzie, and R. C. McPhedran, Transport properties of regular arrays of cylinders, Proc. R. Soc. Lond. A 369(1737), 207-225 (1979).

[37] A. V. Radchik, G. B. Smith, and A. J. Reuben, Quasistatic optical response of separate, touching, and intersecting cylinder pairs, Phys. Rev. B 46(10), 6115-6125 (1992).

[38] L. Rayleigh, On the influence of obstacles arranged in rectangular order upon the properties of a medium, Phil. Mag. 34(211), 481-502 (1892).

[39] A. J. Reuben, A. V. Radchik, and G. B. Smith, The polarizability of chains of touching cylinder pairs, J. Phys. A 26(8), 2021-2036 (1993). 
[40] R. Rodríguez-Ramos, P. Yan, J. C. López-Realpozo, R. Guinovart-Díaz, J. Bravo-Castillero, F. J. Sabina, and C. P. Jiang, Two analytical models for the study of periodic fibrous elastic composite with different unit cells, Compos. Struct. 93(2), 709-714 (2011).

[41] R. Rodríguez-Ramos, H. Berger, R. Guinovart-Díaz, J. C. López-Realpozo, M. Würkner, U. Gabbert, and J. Bravo-Castillero, Two approaches for the evaluation of the effective properties of elastic composite with parallelogram periodic cells, Int. J. Eng. Sci. 58(6), 2-10 (2012).

[42] N. Rylko, Transport properties of a rectangular array of highly conducting cylinders, J. Eng. Math. 38(1), 1-12 (2000).

[43] N. Rylko, Effect of polydispersity in conductivity of unidirectional cylinders, Archives of Materials Science and Engineering 29(1), 45-52 (2008).

[44] J. Segurado, C. González, and J. Llorca, A numerical investigation of the effect of particle clustering on the mechanical properties of composites, Acta. Mater. 51(8), 2355-2369 (2003).

[45] J. D. Thornburg and C. D. Pears, Prediction of the thermal conductivity of filled and reinforced plastics, ASME Paper 65-WA/HT-4 (1965).

[46] S. Torquato, Random Heterogeneous Materials - Microstructure and Macroscopic Properties (Springer-Verlag, New York, 2002).

[47] E. Vilchevskaya and I. Sevostianov, Overall thermal conductivity of a fiber reinforced composite with partially debonded inhomogeneities, Int. J. Eng. Sci. 98, 99-109 (2016).

[48] A. Weil, Elliptic Functions According to Eisenstein and Kronecker (Springer-Verlag, Berlin, 1976).

[49] S. Wolfram, Mathematica Book. 4th Edition (Cambridge University Press, Cambridge, 1999).

[50] P. Yan, J. S. Dong, F. L. Chen, and F. Song, Unified complex variable solution for the effective transport properties of composites with a doubly-periodic array of fibers, ZAMM - Z. Angew. Math. Mech. 97(4), 397-413 (2017).

[51] P. Yan, F. L. Chen, C. P. Jiang, and F. Song, An eigenfunction expansion-variational method in prediction of the transverse thermal conductivity of fiber reinforced composites considering interfacial characteristics, Compos. Sci. Technol. 70(12), 1726-1732 (2010). 